\title{
Intrahepatic Cholestasis of Pregnancy Refractory to Multiple Medical Therapies and Plasmapheresis
}

\author{
Adam John Covach, MD ${ }^{1}$ William Nicholas Rose, MD ${ }^{1}$ \\ ${ }^{1}$ Department of Pathology and Laboratory Medicine, University of \\ Address for correspondence William Nicholas Rose, MD, Department \\ Wisconsin Hospitals and Clinics, Madison, Wisconsin \\ of Pathology and Laboratory Medicine, University of Wisconsin \\ Hospitals and Clinics, Madison, WI (e-mail: wrose@uwhealth.org).
}

Am J Perinatol Rep 2017;7:e223-e225.

\begin{abstract}
Objectives We report on a patient suffering from intractable itching secondary to intrahepatic cholestasis of pregnancy (ICP) unresponsive to conventional medical therapies. She was started on a regimen of therapeutic plasma exchange (TPE), which is often efficacious in relieving patient's itching from all causes of cholestasis, including ICP.

Methods We performed a retrospective review of a patient's medical record.

Results Following initial TPE, the patient reported dramatic relief of her itching and consequent insomnia. However, this effect was short lived, as subsequent TPE provided minimal relief, and may have actually worsened her itching. Out of concern for poor

Keywords

- intrahepatic cholestasis of pregnancy

- plasmapheresis

- bile acid

- hepatitis C fetal outcomes, delivery was induced at 34 weeks gestational age. The child had an uncomplicated neonatal intensive care unit stay following delivery, and the mother reported $>90 \%$ relief of her symptoms 2 weeks after delivery.

Conclusion TPE often provides longer term relief of itching because of ICP; however, it is not a panacea for these symptoms, and sometimes only delivery of the fetus can relieve maternal symptoms. In addition to the refractoriness to TPE, the case is also unusual for the early onset of ICP symptoms and the comorbidity of hepatitis C.
\end{abstract}

Intrahepatic cholestasis of pregnancy (ICP) is a rare complication of pregnancy which manifests as pruritus due to increased bile acids, usually during the third trimester of pregnancy. ${ }^{1}$ Although the etiology of ICP is unknown, it appears that both genetic and environmental factors play a role in its development. ${ }^{2}$

Fetal complications such as prematurity and stillbirth may result; therefore, delivery is often induced as early as possible (i.e., typically 36 weeks). ICP then typically resolves in days to weeks. Temporizing treatment is often aimed at symptom relief by lowering bile acid levels by preventing its absorption with ursodeoxycholic acid (UDCA) or cholestyramine.

Therapeutic plasma exchange (TPE; also known as plasmapheresis) is the replacement of patient plasma with a replacement fluid such as $5 \%$ albumin. A common TPE procedure is called a "one plasma volume" replacement that replaces approximately $63 \%$ of the patient's starting plasma volume. The rationale for using TPE in ICP is to remove bile acid from the plasma which will then cause bile acid to be removed from the

received

February 5, 2017

accepted after revision

September 26, 2017 affected tissues via equilibration between the two compartments. Such an equilibration typically occurs in large part over the 2 days following the procedure.

Direct removal by TPE or other mechanisms can also be used to provide dramatic relief lasting up to 5 days in cases refractory to medical therapies. A case report in 2005 demonstrated that TPE provided substantial and instantaneous relief for a patient with severe ICP that was refractory to medical therapy. ${ }^{3}$ That 2005 case report initially informed us of the potential utility of TPE in ICP.

In addition, other case reports demonstrated that TPE provided symptom relief for pregnant patients with cholestasis due to other causes such as primary biliary cirrhosis ${ }^{4}$ and progressive familial intrahepatic cholestasis. ${ }^{5}$

Women infected with hepatitis $C$ seem to be at increased risk of ICP including a slightly earlier onset of symptoms. ${ }^{6}$ The patient reported here is unusual because rather than an onset at $\sim 23$ to 31 weeks as has been reported previously, her onset of symptoms was 8 weeks.
Copyright @ 2017 by Thieme Medical Publishers, Inc., 333 Seventh Avenue, New York, NY 10001, USA. Tel: +1(212) 584-4662.
License terms

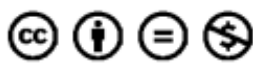

10.1055/s-0037-1609041. ISSN 2157-6998. 


\section{Methods}

We performed a retrospective chart review of the patient's medical record.

\section{Results}

We present a 26-year-old G2P1001, whose previous pregnancy resulted in a full-term vaginal delivery of a healthy girl. She has a history of active hepatitis $\mathrm{C}$ since birth, for which she has failed two previous antiviral regimens secondary to therapy-induced leukopenia. She is not on any medications and is awaiting novel hepatitis $\mathrm{C}$ regimens which do not involve interferon. The patient is otherwise healthy, with no other treated medical conditions.

She had an uncomplicated pregnancy until she started having diffuse itching without visible skin manifestations starting around 8 weeks' gestation. This was soon diagnosed as ICP. She did not have this problem with her first pregnancy 2 years prior. At 15 weeks' gestation, she developed severe insomnia secondary to her itching. The onset of ICP symptoms at such an early gestational age is a relatively rare.

Initial therapeutic recommendations included cold baths, UDCA, and diphenhydramine. She was granted initial relief by these therapies, but her symptoms continued to progress despite optimal medical therapy. Her bile acids were markedly high throughout pregnancy (25-89 $\mu \mathrm{mol} / \mathrm{L})$, despite treatment with cholestyramine and UDCA. Bilirubin and lactate dehydrogenase remained normal, and her aspartate transaminase/

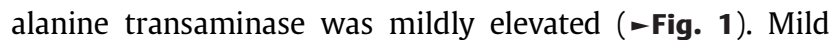
symptomatic relief was reported with UDCA. All other medications were discontinued secondary to side effects and failure to provide significant relief of her symptoms.

As she had failed conventional medical therapy, it was decided to give her a trial of TPE, as this has been reported to provide lasting relief in patients with ICP, lasting up to 5 days. Seven TPEs with $5 \%$ albumin were administered over 4 weeks. The dates were $6 / 17,6 / 24,7 / 1,7 / 8,7 / 11,7 / 15$, and $7 / 1$ (see - Fig. 1). Although the first TPE did not totally relieve her itching, it made it bearable. These effects initially lasted about 2 days; therefore, TPE was planned for twice a week until her initially scheduled delivery at 36 weeks' gestation. Later TPEs provided minimal relief and, per patient reports, may have actually worsened her itching. As a result, TPE was discontinued at 32 weeks' gestation.

Due to the progressive nature of her symptoms and out of concern for possible complications to her child, her planned delivery was bumped up to 34 weeks. Although the initial recommendation before TPE was to use TPE as a bridge to delivery at 36 to 37 weeks, extensive counseling and collaboration led to a revised plan of 34 weeks with betamethasone. The main factor was the concern for increased risk of unpredictable fetal demise due to both the magnitude of bile acid level and the rapid rate of increase of bile acid levels despite multiple therapies. In the interim, she was able to get some mild relief of her pruritus and insomnia with continued UDCA use and zolpidem, respectively.

She had an uncomplicated delivery induced at 34 weeks, and reported $90 \%$ relief of symptoms at 2 weeks postpartum. Her child had an uncomplicated neonatal intensive care unit stay for observation with no evidence of sequelae from premature delivery.

\section{Discussion}

Case reports and series have demonstrated that TPE often provides dramatic relief of itching and related insomnia in patients who have cholestatic disease refractory to standard medical management, regardless of the cause of cholestasis. This effect usually lasts for 4 to 5 days. Our patient never received quite the dramatic symptomatic improvement reported in these case reports, and what little effect she did received lasted at best, 1 to 2 days.

In addition, her bile acids level remained elevated despite treatment with both TPE and oral medications. This fact underscores that TPE is not a cure-all of ICP. Its place as a second-line therapy is secondary to multiple factors, including cost, potential exposure to donors if plasma is used as a replacement fluid, and general effectiveness of easier to use oral medications. We speculate that the comorbidity of hepatitis $C$ may have contributed to the early onset of symptoms and the refractoriness to TPE, but we do not immediately have an explanation, mechanism, causality, or interaction.

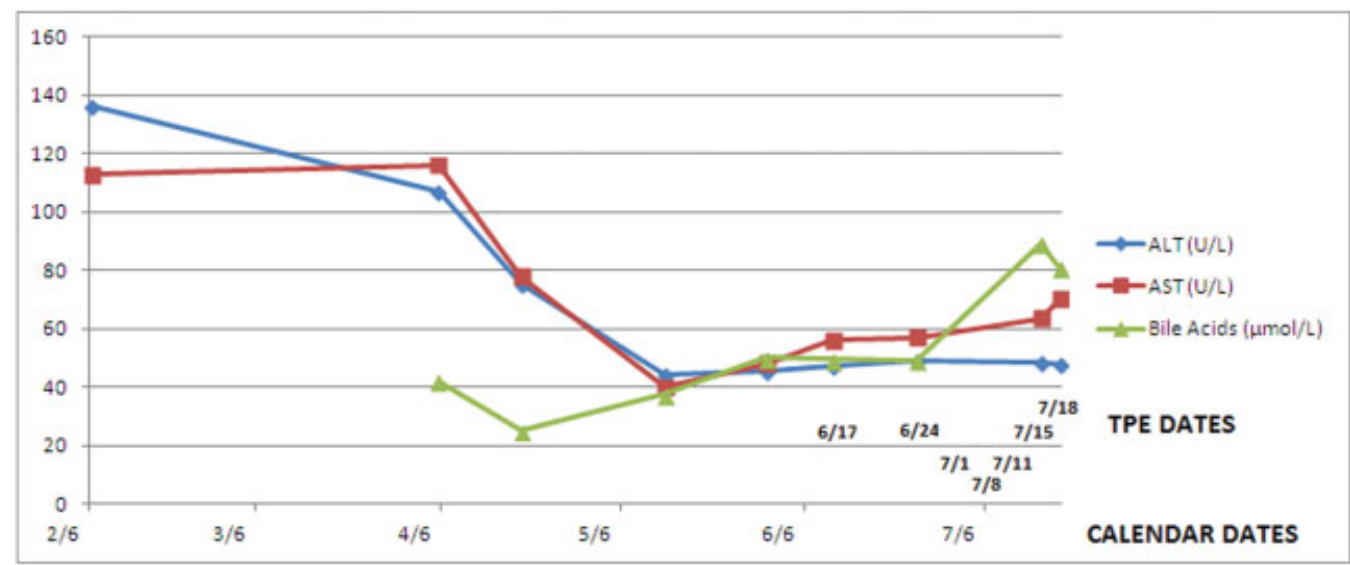

Fig. 1 Serum ALT, AST, and bile acid levels along with dates of TPE. ALT, alanine transaminase; AST, aspartate transaminase; TPE, therapeutic plasma exchange. 
We also speculate that charcoal administration could be a potential adjunct therapy. This is based on a report of charcoal being associated with a significant decrease in bile acid levels but not symptom relief. ${ }^{7}$ We speculate that including charcoal as part of a combination of therapies might further chip away at the bile acid level and might provide a decrement that would be sufficient to go past the threshold for noticeable symptom relief. On the contrary, the likely side effects of charcoal may cause patients to refuse a therapy that has not been proven to provide consistent symptom relief.

The most feared adverse outcome of ICP is fetal demise; however, the reasons this happens are currently unknown. About $90 \%$ of cases of fetal demise secondary to ICP occur after 37 weeks; therefore, inducing delivery prior to 40 weeks is a sensible option for many patients with relatively treatable ICP. Delivery earlier than 37 weeks is often considered in the face of disease refractory to therapy or a prior history of fetal demise in the setting of ICP. The current consensus is delivery at 36 weeks for such patients. Patients who have experienced ICP in one pregnancy have a $45 \%$ chance of experiencing this complication in future pregnancies; therefore, patients should be informed of this in a larger discussion of potential future pregnancies.

Disclosure

This case report was presented as an abstract poster at the American Society for Apheresis 2014 Annual Meeting in San Francisco, CA. ${ }^{8}$

\section{Conflict of Interest}

The authors have no financial or other conflict of interest to report.

\section{References}

1 Lindor K, Lee R. Intrahepatic cholestasis of pregnancy. In: Angulo P, Lockwood C, Travis A, Barss V, eds. UpToDate, Waltham, MA; 2013

2 Webb GJ, Elsharkawy AM, Hirschfield GM. The etiology of intrahepatic cholestasis of pregnancy: towards solving a monkey puzzle. Am J Gastroenterol 2014;109(01):85-88

3 Warren JE, Blaylock RC, Silver RM. Plasmapheresis for the treatment of intrahepatic cholestasis of pregnancy refractory to medical treatment. Am J Obstet Gynecol 2005;192(06):2088-2089

4 Alallam A, Barth D, Heathcote EJ. Role of plasmapheresis in the treatment of severe pruritus in pregnant patients with primary biliary cirrhosis: case reports. Can J Gastroenterol 2008;22(05): 505-507

5 Mathias A, Wax JR, Pinette MG, Cartin A, Blackstone J. Progressive familial intrahepatic cholestasis complicating pregnancy. J Matern Fetal Neonatal Med 2009;22(09):816-818

6 Paternoster DM, Fabris F, Palù G, et al. Intra-hepatic cholestasis of pregnancy in hepatitis $C$ virus infection. Acta Obstet Gynecol Scand 2002;81(02):99-103

7 Kaaja RJ, Kontula KK, Räihä A, Laatikainen T. Treatment of cholestasis of pregnancy with peroral activated charcoal. A preliminary study. Scand J Gastroenterol 1994;29(02):178-181

8 Covach AJ, Rose WN. Intrahepatic cholestasis of pregnancy refractory to multiple medical therapies and plasmapheresis. J Clin Apher 2014;29(01):58 